$$
\text { CONF-970159--2 }
$$

\title{
THERMAL DIFFUSIVITY MAPPING OF 4D CARBON-CARBON COMPOSITES
}

H. Wang and R. B. Dinwiddie

High Temperature Materials Laboratory, Metals and Ceramics Division

Oak Ridge National Laboratory

Oak Ridge, TN 37831-6064

\section{ABSTRACT}

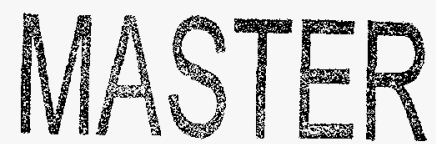

High resolution, 2-D thermal diffusivity maps of carbon-carbon composites were obtained by a state-of-the-art infrared thermal imaging system. Unlike the traditional single-point IR detector used for thermal diffusivity measurements, the IR camera is capable of capturing images in its $256 \times 256$ pixel Focal Plane Array detector in a snap-shot mode. The camera takes up to 200 images at a rate of 120 frames/second. The temperature resolution of the IR camera is $0.015^{\circ} \mathrm{C}$ and the spatial resolution is $20 \mu \mathrm{m}$. Thermal diffusivity was calculated for each pixel. Four-direction carbon-carbon composites were used for the thermal diffusivity mapping study. The fiber bundles along the heat flow direction were found to have $25 \%$ higher diffusivity values than the surrounding matrix. The diffusivity map also showed detailed local variations in diffusivity which were impossible to measure using a single-point detector. Accurate diffusivity maps are very important to the design of composite materials.

\section{INTRODUCTION}

Composite materials, such as carbon-carbon composites, have been used as structural components for high-temperature applications. One of the important characteristics of a composite is its thermal transport property, i.e. thermal conductivity for steady state heat conduction or thermal diffusivity for transient heat conduction. Accurate measurements of thermal transport properties are crucial to the component design. Thermal diffusivity[1] is the most often used technique for determination of transport properties since thermal conductivity, $\mathrm{K}$, can be calculated from the product of density, $\rho$, specific heat, $C_{p}$, and thermal diffusivity,

\section{MISTAETTOY OF THS NOQUMENT IS UNLMITE}

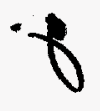




\section{DISCLAIMER}

This report was prepared as an account of work sponsored by an agency of the United States Government. Neither the United States Government nor any agency thereof, nor any of their employees, make any warranty, express or implied, or assumes any legal liability or responsibility for the accuracy, completeness, or usefulness of any information, apparatus, product, or process disclosed, or represents that its use would not infringe privately owned rights. Reference herein to any specific commercial product, process, or service by trade name, trademark, manufacturer, or otherwise does not necessarily constitute or imply its endorsement, recommendation, or favoring by the United States Government or any agency thereof. The views and opinions of authors expressed herein do not necessarily state or reflect those of the United States Government or any agency thereof. 


\section{DISCLAIMER}

Portions of this document may be illegible in electronic image products. Images are produced from the best available original document. 
$\alpha$ : that is, $K=\alpha \rho C_{p}$. The flash diffusivity technique is an ASTM standard material testing procedure[2]. Data analysis algorithms developed by Clark and Taylor[3] and Koski[4] are often used to calculate thermal diffusivity.

The traditional flash diffusivity technique utilizes a single-point infrared detector. It measures the average temperature rise at the back surface of a specimen after a heat pulse. This technique requires the specimen to be homogeneous and the heating of the specimen to be uniform. Most solid state materials with the exception of composites can meet these requirements.

Composites can be divided into three basic groups: dispersed, layered and fiber-reinforced composites. Thermal diffusivity of dispersed composites can be measured by the flash technique as long as the specimen is thick enough to represent the bulk material. Layered composites have been studied extensively for many years [5-7]. Thermal diffusivity of two to four layered materials can be calculated from various one-dimensional heat flow models. Fiber-reinforced composites have more complex structures and the thermal diffusivity measurements are less straightforward. Fibers can be continuous or chopped and the stacking of the fibers can be periodic or random. Taylor[8] showed at least four unit cells are needed to get an accurate measurement for composites having periodic structures. In the case of aligned fibers, the volume percentage of the fibers must be very low in order to get a good coupling effect between the fibers and the matrix. In continuous fiber ceramic composites, the thermal transport property of the fibers may be very different from the matrix and the fiber volume can be high. Heat conduction can change significantly depending on the relative orientation between the heat flow and the fibers.

Thermal diffusivity of fiber-reinforced composites has been measured using the traditional flash technique. Some researchers have developed models to estimate the thermal diffusivity of fiber reinforced composites[9-12]. Accurate thermal transport properties of both the matrix and the fibers must be known in order to apply the rule-of-mixtures. However, thermal properties of the material may change after processing and sintering. It is very important to be able to measure thermal diffusivity of different phases in a composite. Recently, thermal diffusivity imaging of CFCC was studied by Ahuja et al[13]. They used an IR camera to generate thermal diffusivity maps and studied artificial defects of at least 4 $\mathrm{mm} \times 4 \mathrm{~mm}$ in dimension.

In the present study, a high-resolution infrared camera was used to create thermal diffusivity maps of carbon-carbon composites. Unlike the single-point IR detector, the camera has a $256 \times 256$ pixel InSb Focal Plane Array (FPA). Each pixel in the FPA is an individual IR detector. The camera can operate in a snapshot mode where all the pixels are exposed to IR radiation simultaneously for the same amount of time. This feature allows the camera to be used as a 2-D IR detector in the flash technique. The camera can take up to 200 images at a rate of 
$120 \mathrm{~Hz}$. Thermal diffusivity of each pixel was calculated and formed a thermal diffusivity map with $20 \mu \mathrm{m}$ spatial resolution.

Four-direction (4D) carbon-carbon composites made by Fiber Materials Inc. with Amoco Performance Products P-25 fibers were tested in this study. A preliminary study[14] showed the possibility of generating diffusivity maps for the carbon-carbon composites. More powerful image analyzing software and better spatial resolution were utilized in the present study.

\section{EXPERIMENTAL}

An infrared camera manufactured by Amber, Goleta $\mathrm{CA}$, was used as the detector described in the flash technique. Figure 1 shows the experimental setup for diffusivity mapping. A typical specimen was a disk about $12.5 \mathrm{~mm}$ in diameter. It was placed in a stainless steel sample holder and mounted in front of a high power xenon flash lamp. A low thermal conductivity clay was used to hold the specimen and minimize heat loss via the sample holder.

The IR camera monitored the back surface of the specimen. A photo diode was used to detect the flash and send a trigger signal to the control workstation. The camera took 200 images at a rate of $120 \mathrm{~Hz}$ following the trigger. The images were stored in the computer. An Excel ${ }^{\mathrm{TM}}$ Macro program was written to calculate thermal diffusivity pixel by pixel. Spatial resolution of $20 \mu \mathrm{m}$ was used $(7.5 \mu \mathrm{m}$ resolution can be achieved using a microscope lens with a field of view of $1 \mathrm{~mm} \times 1$ $\mathrm{mm}$ ). Parker's technique[1] was used to calculate the thermal diffusivity. It assumes no heat loss and measures the time for the back surface to reach half of the maximum temperature, $t_{0.5}$. Knowing the specimen thickness, $d$, thermal diffusivity is simply calculated as:

$$
\alpha=0.1388 \mathrm{~d}^{2} / \mathrm{t}_{0.5}
$$

where $\alpha$ is the thermal diffusivity in $\mathrm{cm}^{2} / \mathrm{s}$. The results were stored in a spreadsheet and can be easily plotted by graphic software packages.

A 4D coarse weaved carbon-carbon composite sample, from Fiber Materials Inc., $12.5 \mathrm{~mm}$ in diameter and $14 \mathrm{~mm}$ thick was used for thermal diffusivity mapping. There were 4000 fibers in a bundle. The composite was produced from P-25 pitch precursor carbon fibers woven in three directions in the horizontal plane, 120 degrees apart with the fourth fiber direction perpendicular to the plane. The overall fiber volume was $48 \%$. The carbon fiber preform was densified through a series of repetitive impregnation, pressure carbonization and graphitization cycles until a density of $2.02 \mathrm{~g} / \mathrm{cm}^{3}$ was achieved. The inpregant was a coal tar based pitch material manufactured by Allied Signal which infiltrated the spaces between the 


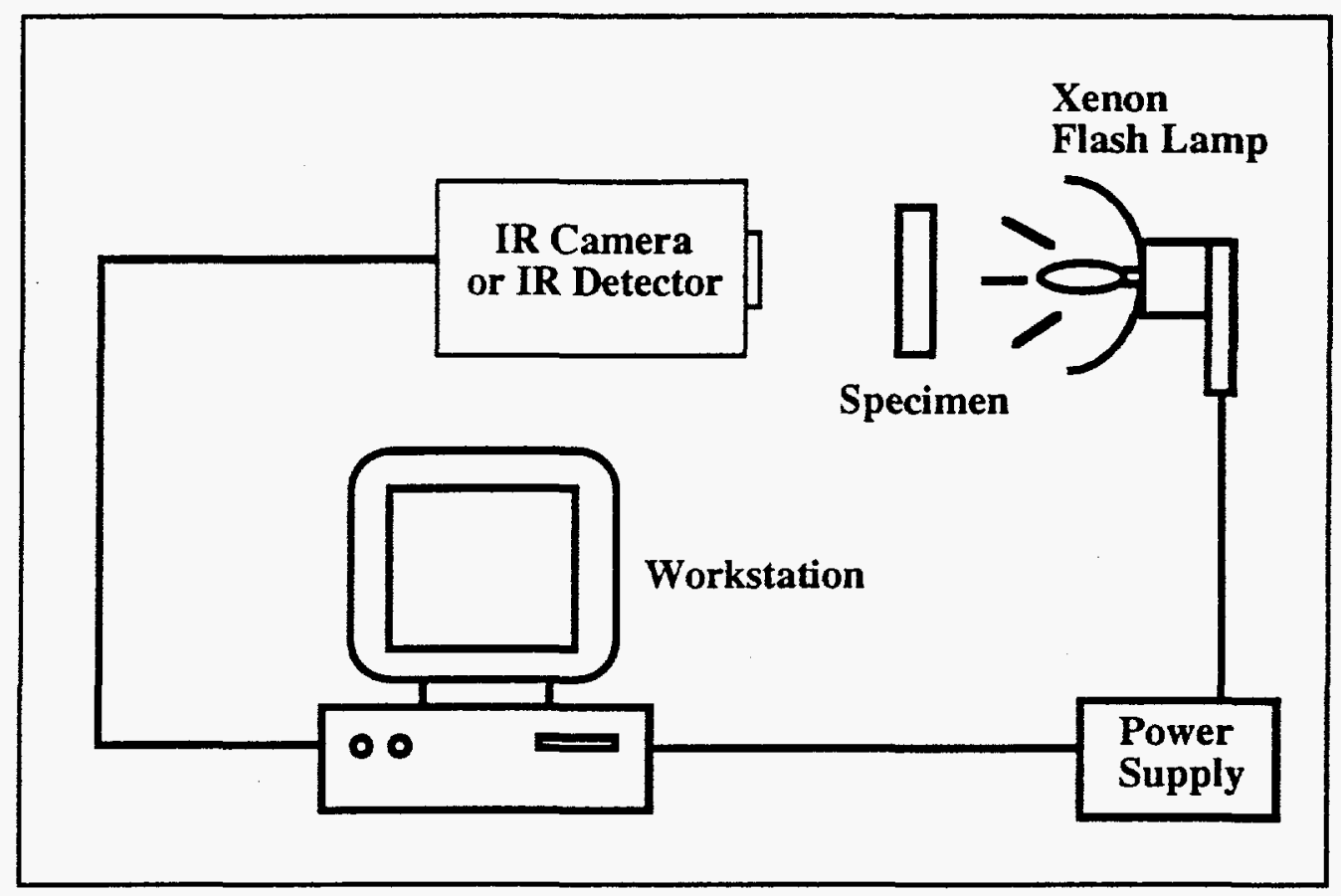

Figure 1. Flash diffusivity measurement using an IR detector or camera.

fibers as a liquid, and was converted to pure carbon by heating to $650^{\circ} \mathrm{C}$. The resulting carbon reinforced carbon block was heat treated at $2250^{\circ} \mathrm{C}$ to graphitize the carbon. The specimen was cut parallel to the horizontal planes. The direction of heat flow was parallel to the fourth set of fiber bundles.

\section{RESULTS AND DISCUSSION}

The rapid heating of carbon-carbon composite specimens was captured by the IR camera. Figure 2 is an IR image 32 milliseconds after the heat pulse. The background was subtracted to reduce the noise level. The fiber bundles in the same direction as the heat flow showed higher temperatures which indicated they heated up faster than the matrix and fiber bundles in the horizontal planes. The high temperature sensitivity of the camera also showed temperature differences between the matrix materials and the horizontal fiber bundles. The horizontal fiber bundles showed slightly higher heating than the matrix. An intensity histogram of 65,536 pixels in the same image is shown in Figure 3. The first peak at zero intensity comes from the black area representing the sample holder. The second broad peak at intensity level 16 represents the matrix and horizontal fiber bundles. A third peak 


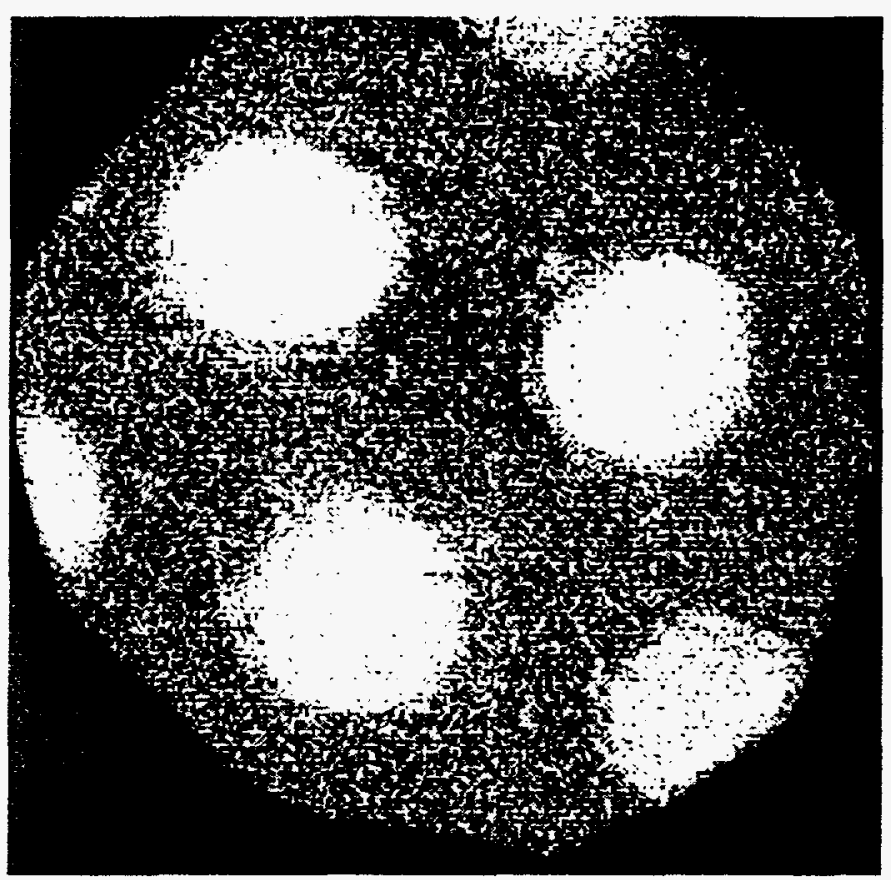

Figure 2. IR image $32 \mathrm{~ms}$ after the heat pulse.Fiber bundles are $1.5 \mathrm{~mm}$ in diameter.

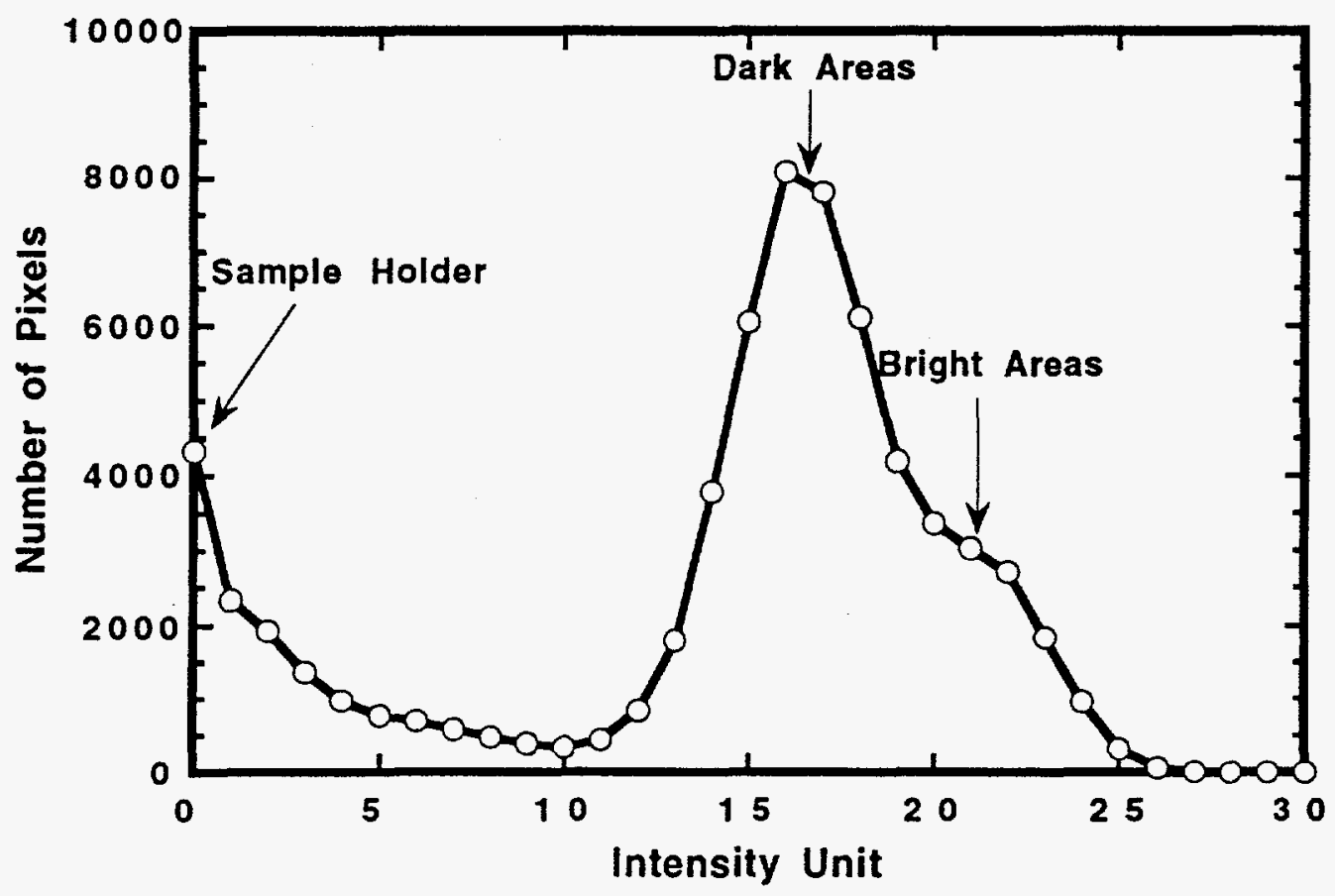

Figure 3. Intensity histogram of Figure 2. Three peaks can be found in the plot. 


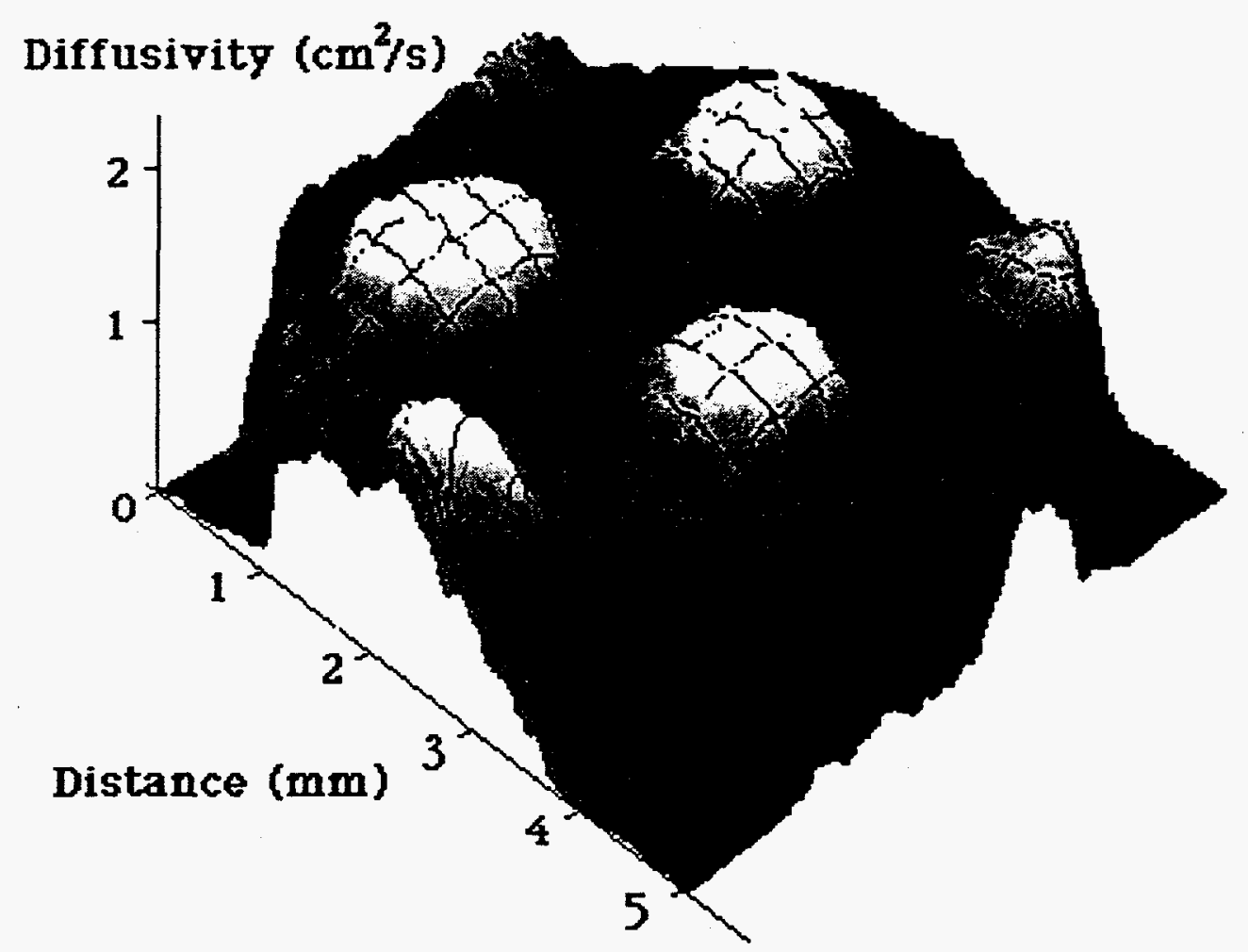

Figure 4. Thermal diffusivity map of a 4D carbon-carbon composite. The grey matrix area has an average diffusivity of $1.20 \mathrm{~cm}^{2} / \mathrm{s}$ and the bright fiber bundles have average diffusivity of $1.50 \mathrm{~cm}^{2} / \mathrm{s}$. The black edge is the sample holder.

at intensity level 22 appeared as a shoulder. It represents the bright fiber bundles parallel to the heat flow.

Using the whole sequence of 200 images, thermal diffusivity values of each pixel were calculated. A $256 \times 256$ pixel thermal diffusivity map is shown as a surface plot in Figure 4. Thermal diffusivity of the fiber bundles in the same direction as the heat flow averages $1.50 \pm 0.17 \mathrm{~cm}^{2} / \mathrm{s}$ and the average thermal diffusivity of the fiber bundles in the horizontal plane and the matrix was $1.20 \pm$ $0.15 \mathrm{~cm}^{2} / \mathrm{s}$. Averaging was done via the imaging software. The average thermal diffusivity of the entire specimen was $1.30 \mathrm{~cm}^{2} / \mathrm{s}$.

The same $14 \mathrm{~mm}$ thick specimen was also tested using a single-point $\mathrm{InSb}$ infrared detector. The measured half rise time was $132 \pm 5 \mathrm{~ms}$ and the thermal diffusivity calculated using Parker's method[1] was $1.52 \pm 0.01 \mathrm{~cm}^{2} / \mathrm{s}$. This result 
indicated that the average thermal diffusivity measured by the single-point detector tends to be closer to the high conductivity fiber bundle value. Therefore, it did not represent the true average thermal diffusivity value of the specimen. The singlepoint IR detector would respond first to the temperature rise of the fiber bundles and subsequently to the horizontal fiber bundles and the matrix. Thus the singlepoint detector output is a summation of several rise and fall signals with the fastest and highest rise due to the fiber bundles parallel to the direction of heat flow. The high fraction of the fiber bundle in the sample, nearly $25 \%$, may contribute to the high diffusivity value. The thermal diffusivity map provided more information on the carbon-carbon composite than a single diffusivity value. The coupling effect between the matrix and the fiber bundle showed as a gradual change in diffusivity at the interface. The center of the fiber bundles showed the highest diffusivity.

The as-received P-25 fibers had low thermal conductivity, $25 \mathrm{~W} / \mathrm{mK}$, to start with. During high temperature graphtization the microstructure of the fibers changed and thermal conductivity increased dramatically. The fiber bundles in the 4D carbon-cabbon composite had thermal conductivities ranging from 170 to 200 $\mathrm{W} / \mathrm{mK}$. The carbon-carbon composite was chosen as an example to demonstrate the thermal diffusivity mapping technique. The ability to generate detailed thermal diffusivity maps will certainly be beneficial to the design of high temperature materials.

\section{SUMMARY}

Thermal images of heat conduction through 4D carbon-carbon composites were captured by an IR camera. Fiber bundles orientaed parallel to the heat flow were found conducting heat faster than the matrix. A thermal diffusivity map of the 4D carbon-carbon composite was generated. This technique has shown promising applications in characterizing thermal transport properties of composite materials.

\section{ACKNOWLEDGMENT}

This work was supported by the U.S. Department of Energy, Assistant Secretary for Energy Efficiency and Renewable Energy, Office of Transportation Technologies, as part of the High Temperature Materials Laboratory User Program under contract DE-AC05-96OR22464, managed by Lockheed Martin Energy Research Corporation. This research work was also supported in part by a Postdoctoral Research Associates Program administered jointly by ORISE and ORNL. 


\section{REFERENCES}

[1] W. J. Parker, R. J. Jenkins, C.P. Butler and G.L. Abbott, "Flash Method of Determining Thermal Diffusivity, Heat Capacity and Thermal Conductivity", J. Appl. Phy. 32(9) 1679 (1961)

[2] ASTM Designation: E 1461 -92, "Standard Test Method for Thermal Diffusivity of Solids by the Flash Method", Annual Book of ASTM Standards, Vol. 14.02, 933-940 (1992)

[3] L.M. Clark and R.E. Taylor, "Radiation Loss in the Flash Method for Thermal Diffusivity", J. Appl. Phys., Vol.46 No.2, 714-719 (1975)

[4] J. A. Koski, "Improved Data Reduction Methods for Laser Pulse Diffusivity Determination with the Use of Minicomputers", Proceeding of the 8th Symposium of Thermophysical Properties, Vol. II: 94-103 (1981)

[5] C.S. Ang, H.S. Tan and S.L. Chan, "Three-layer Thermal Diffusivity Problem Applied to Measurements on Mercury", J. Appl. Phys., Vol.44 No.2, 687-691 (1973)

[6] R.F. Bulmer and R. Taylor,"Measurement by the Flash Method of Thermal Diffusivity in Two-layer Composite Samples", High Temperatures-High Pressures, Vol. 6 491-497 (1974)

[7] T. Ishiguro, A. Makino, N. Araki and N. Noda, "Transient Temperature Response in Functionally Gradient Materials", International Journal of Thermophysics, Vol. 14 No. 1 101-121 (1993)

[8] R. E. Taylor, J. Jortner and H. Groot, "Thermal Diffusivity of Fiber-reinforced Composites Using Laser Flash Technique", Carbon 23, 215-222 (1985)

[9] K.K. Chawla, "Ceramic Matrix Composites", Chapman and Hall, 216-218 (1993)

[10] E. Behren, "Thermal Conductivity of Composite Materials", J. Composite Materials, (2)2 1968

[11] D.P.H. Hasselman and L.F. Johnson, J. Composite Materials, 27 (508) 1987

[12] H. Bhatt, K.Y. Donaldson, D.P.H. Hasselman and R.T. Bhatt, "Effect of Fiber Orientation on the Thermal Conductivity of a Uniaxial Carbon-reinforced Aluminoborosilicate Glass-matrix Composite for Various Specimen Geometries", J. Mater. Sci. (27) 6653 (1992)

[13] S. Ahuja, W.A. Ellingson, J. S. Steckenrider and S. King, "Thermal Diffusivity Imaging of Continuous-Fiber Ceramic Composite (CFCC) Materials and Components", Thermal Conductivity 23, Ed. by R.B. Dinwiddie et al., published by Technomic, 311-321 (1996)

[14] H.Wang and R.B. Dinwiddie, "Advanced Thermal Imaging of Composite Materials", Advances in Ceramic-Matrix Composites III, Ceramic Transactions Vol. 74, (1996) 